\title{
Cardiac involvement in cystic fibrosis evaluated using cardiopulmonary magnetic resonance
}

\author{
Jakub Lagan ${ }^{1,2}$. Josephine H. Naish ${ }^{2}$. Joshua Bradley ${ }^{1,2}$. Christien Fortune ${ }^{1,2}$. Charlie Palmer ${ }^{1}$ David Clark ${ }^{1}$. \\ Erik B. Schelbert ${ }^{3,4,5}$. Matthias Schmitt ${ }^{1,2} \cdot$ Rowland Bright-Thomas $^{1,2} \cdot$ Christopher A. Miller $^{1,2,6}$
}

Received: 29 July 2021 / Accepted: 11 December 2021 / Published online: 7 January 2022

(c) The Author(s) 2022

\begin{abstract}
Cystic fibrosis (CF) transmembrane conductance regulator is expressed in myocardium, but cardiac involvement in CF remains poorly understood. The recent development of a combined cardiopulmonary magnetic resonance imaging technology allows for a simultaneous interrogation of cardiac and pulmonary structure and function. The aim of this study was to investigate myocardial manifestations in adults with CF, both in a stable state and during an acute respiratory exacerbation, and to investigate the relationship between cardiac and pulmonary disease. Healthy adult volunteers $(\mathrm{n}=12)$ and adults with $\mathrm{CF}(\mathrm{n}=10)$ were studied using a multiparametric cardiopulmonary magnetic resonance protocol. CF patients were scanned during an acute respiratory exacerbation and re-scanned when stable. Stable CF was associated with left ventricular dilatation and hypertrophy (LVH; left ventricular mass: CF $59 \pm 9 \mathrm{~g} / \mathrm{m}^{2}$ vs. control $50 \pm 8 \mathrm{~g} / \mathrm{m}^{2} ; \mathrm{p}=0.028$ ). LVH was predominantly driven by extracellular myocardial matrix expansion (extracellular matrix mass: CF $27.5 \pm 3.4 \mathrm{~g}$ vs. control $23.6 \pm 5.2 \mathrm{~g}$; $\mathrm{p}=0.006$; extracellular volume [ECV]: CF 27.6 [24.7-29.8]\% vs. control 24.8 [22.9-26.0]\%; $\mathrm{p}=0.030$ ). Acute CF was associated with an acute reduction in left ventricular function (ejection fraction: acute $57 \pm 3 \%$ vs. stable $61 \pm 5 \% ; p=0.025$ ) and there was a suggestion of myocardial oedema. Myocardial oedema severity was strongly associated with the severity of airflow limitation $(\mathrm{r}=-0.726, \mathrm{p}=0.017)$. Multiparametric cardiopulmonary magnetic resonance technology allows for a simultaneous interrogation of cardiac and pulmonary structure and function. Stable CF is associated with adverse myocardial remodelling, including left ventricular systolic dilatation and hypertrophy, driven by myocardial fibrosis. CF exacerbation is associated with acute myocardial contractile dysfunction. There is also a suggestion of myocardial oedema in the acute period which is related to pulmonary disease severity.
\end{abstract}

Keywords Cystic fibrosis $\cdot$ Cardiac magnetic resonance $\cdot$ Myocardial fibrosis $\cdot$ Myocardial inflammation $\cdot$ Parametric mapping

Christopher A. Miller

Christopher.Miller@manchester.ac.uk

1 Manchester University NHS Foundation Trust, Wythenshawe Hospital, Southmoor Road, Wythenshawe, Manchester M23 9LT, England, UK

2 Division of Cardiovascular Sciences, School of Medical Sciences, Faculty of Biology, Medicine and Health, Manchester Academic Health Science Centre, University of Manchester, Oxford Road, Manchester M13 9PL, England, UK

3 Department of Medicine, University of Pittsburgh School of Medicine, Pittsburgh, PA, USA
4 UPMC Cardiovascular Magnetic Resonance Center, Heart and Vascular Institute, Pittsburgh, PA, USA

5 Clinical and Translational Science Institute, University of Pittsburgh, Pittsburgh, PA, USA

6 Wellcome Centre for Cell-Matrix Research, Division of Cell-Matrix Biology \& Regenerative Medicine, School of Biology, Faculty of Biology, Medicine \& Health, Manchester Academic Health Science Centre, University of Manchester, Oxford Road, Manchester M13 9PT, England, UK 


$\begin{array}{ll}\text { Abbreviations } & \\ \text { CF } & \text { Cystic fibrosis } \\ \text { CFTR } & \begin{array}{l}\text { Cystic fibrosis transmembrane conduct- } \\ \text { ance regulator }\end{array} \\ \text { DCE Imaging } & \text { Dynamic contrast enhanced imaging } \\ \text { ECV } & \text { Extracellular volume } \\ \text { EF } & \text { Ejection fraction } \\ \text { FEV } & \text { Forced expiratory volume in one second } \\ \text { GBCA } & \text { Gadolinium based contrast agent } \\ \text { hsTnI } & \text { High sensitivity troponin I } \\ \text { K }^{\text {trans }} & \text { Transfer constant (capillary permeability) } \\ \text { LGE } & \text { Late gadolinium enhancement } \\ \text { LV } & \text { Left ventricle } \\ \text { MRI } & \text { Magnetic resonance imaging }\end{array}$

\section{Introduction}

Cystic fibrosis $(\mathrm{CF})$, the most common lethal autosomal recessive disorder in Caucasian populations, is a multisystem condition caused by mutations in the CF transmembrane conductance regulator (CFTR) gene [1]. The resulting impaired transport of chloride and other ions leads to hyperviscous secretions. Pulmonary manifestations remain the main cause of morbidity and mortality and forced expiratory volume in one second $\left(\mathrm{FEV}_{1}\right)$ is the strongest indicator of prognosis [1].

CFTR is expressed in myocardium [2], but while cor pulmonale is well recognised, the heart is not generally considered part of the primary disease expression. Existing data regarding cardiac manifestations are conflicting and it is unclear whether cardiac abnormalities, if present, are related to disease severity [3-5]. With improving life expectancy, understanding cardiac involvement in $\mathrm{CF}$ is important in terms of quality of life, prognosis and transplant candidacy [6].

Magnetic resonance imaging (MRI) can provide unique in vivo insight into myocardial and lung injury [7]. Myocardial cine imaging allows for cardiac functional analysis [8]. Late gadolinium enhancement imaging (LGE) has been extensively validated in detecting focal replacement myocardial fibrosis [9]. Myocardial parametric mapping, including T1, T2 and extracellular volume (ECV) mapping, provides a robust assessment of tissue composition, specifically a free water content (i.e. oedema) and the degree of extracellular collagen deposition (i.e. focal and diffuse fibrosis) [10]. ECV is a well validated tool to measure the extracellular matrix fraction and as such can be used to differentiate the cellular from extracellular left ventricular mass [10]. Pulmonary T1 mapping was shown to detect pulmonary inflammation and pulmonary tissue destruction and fibrosis [11-13]. Dynamic contrast enhanced MRI (DCE-MRI) with contrast agent kinetics modelling allows for the assessment of pulmonary perfusion and capillary permeability $\left(\mathrm{K}^{\text {trans }}\right.$; transfer constant), a surrogate marker of inflammation [14-16].

This study aimed to investigate myocardial manifestations of $\mathrm{CF}$, during the acute exacerbation and in the stable state of the disease, and to analyse the relationship between cardiac and pulmonary disease in CF using a combined cardiopulmonary MRI technology.

\section{Material and methods}

\section{Study design}

This prospective research study aimed to apply a combined cardiopulmonary MRI protocol in healthy volunteers and, for the first time, in patients with CF during an acute pulmonary exacerbation and in a stable period in order to investigate the relationship between cardiac and pulmonary disease.

\section{Study population}

Consecutive consenting adult patients with genetically confirmed CF admitted to Manchester Adult CF Centre, Manchester University NHS Foundation Trust with acute respiratory exacerbation were prospectively recruited [17]. Exclusion criteria included: any history of cardiac disease, contraindication to MRI, glomerular filtration rate $<40 \mathrm{ml} /$ $\mathrm{min} / 1.73 \mathrm{~m}^{2}$. Healthy volunteers with no cardiovascular symptoms, no history of medical conditions and normal electrocardiogram (ECG) were prospectively recruited to act as controls.

\section{Study procedures}

Healthy volunteers underwent blood sampling (blood count, renal function, c-reactive protein (CRP), high sensitivity troponin I (hsTnI)), ECG and MRI.

CF patients underwent identical blood sampling, ECG and MRI and additional measurement of spirometry (EasyOne portable spirometer; NDD Medical technologies, Zurich, Switzerland) and blood gas analysis during the acute hospital admission (acute CF). Investigations were repeated when patients were deemed clinically stable, a minimum of 5 weeks following the acute admission (stable CF). Findings were compared with the healthy volunteers.

\section{Combined cardiopulmonary MRI}

MRI was performed at $1.5 \mathrm{~T}$ scanner (Avanto, Siemens Medical Imaging). Scan duration was $60 \mathrm{~min}$. The MRI protocol is described in the supplementary material and shown in Supplementary Fig. 1. It was also previously described in 
detail [7]. In brief, it included: myocardial steady-state free precession cine imaging, myocardial and pulmonary native T1 mapping, myocardial T2 mapping, myocardial and pulmonary DCE-MRI, post contrast myocardial T1 mapping and LGE imaging.

\section{MRI analysis}

MRI analysis (performed by JL and CAM; both holding level 3 cardiovascular MRI accreditation; Society for Cardiovascular Magnetic Resonance) is described in the supplementary material and was also described previously in detail [7]. In brief, cardiac volumetric analysis was performed using Circle CVI42 (Circle Cardiovascular Imaging, Canada) according to guidelines [8]. Parametric maps were analysed in Horos (Horos2K v2.2.0 The Horos Project). Myocardial ECV was calculated using same-day haematocrit as described previously [18]. LV extracellular matrix mass (g) was calculated by multiplying LV mass by ECV [10]. LV cellular mass (g) was calculated by multiplying LV mass by (100\%-ECV). DCE imaging was analysed using a custom written Matlab code (v9.0, The MathWorks, USA). Contrast agent kinetics were modelled using an extended version of a Kety model to calculate myocardial and pulmonary capillary permeability $\left(\mathrm{K}^{\text {trans }}\right)$ and pulmonary extracellular volume fraction (Ve) [14]. Pulmonary blood flow (F) was calculated by deconvolution of the first pass dynamic data as described previously $[14,15]$.

\section{Statistical analysis}

This was the first study to apply cardiac MRI in CF thus there were no data to base a power calculation upon. Using data from other populations, $10 \mathrm{CF}$ patients were required to detect an absolute minimum difference, between acute and stable scans, of $2 \%$ in terms of absolute change in ECV, with $80 \%$ power at a $5 \%$ significance level (2-sided), assuming a standard deviation of within-patient differences equal to $2 \%$ [18].

Data are summarised using mean and standard deviation or median and interquartile range, and compared using $t$ tests or non-parametric equivalents, as appropriate. Correlation analysis was performed using Pearson or Spearman correlation. $P$ value of $\leq 0.05$ was considered significant. Analyses were performed using SPSS (v22, IBM).

\section{Results}

\section{Participants characteristics}

Ten CF patients with an acute respiratory exacerbation were recruited; median age $28(25-36)$ years; $2(20 \%)$ female. Baseline characteristics are summarised in Table 1. Mean admission arterial partial pressure of oxygen was $8.9 \pm 0.9 \mathrm{kPa}$ and $\mathrm{FEV}_{1}$ was $1.4 \pm 0.4 \mathrm{~L} / \mathrm{s}$. Details of antibiotic treatment are provided in Supplementary Material. Median interval from admission to acute MRI scan was 12 (8-16) days. No patient had detectable hsTnI. Twelve healthy volunteers were recruited as controls; median age 37 (27-45) years; 6 (50\%) female; Table 1.

Nine CF patients underwent MRI in the stable stage (one became too unwell to re-attend). Median time from admission to stable MRI was 85 (52-398) days. Mean FEV ${ }_{1}$ was $1.7 \pm 0.5 \mathrm{~L} / \mathrm{s}$. Five patients had severe disease (percentage of predicted $\left.\mathrm{FEV}_{1}\left(\mathrm{FEV}_{1} \%\right)<40 \%\right)$ and 5 had moderate disease $\left(\mathrm{FEV}_{1} \% 40 \%-69 \%\right)$. All spirometry indices improved between acute and stable assessments. CRP and white cell levels improved although these remained elevated compared to controls.

\section{Myocardial manifestations of CF}

Acute CF exacerbation was associated with a significant reduction in left ventricular ejection fraction (LVEF), mediated by an increase in LV end systolic volume (control LVEF $63 \pm 5 \%$ vs. acute CF LVEF $57 \pm 3 \%, p=0.004$; Table 2; Fig. 1). LVEF improved significantly in the stable period (stable CF LVEF $61 \pm 5 \% ; p=0.025$ compared to acute CF) such that it was no different to that in controls $(p=0.27)$. Acute CF exacerbation was also associated with a significant reduction in right ventricle ejection fraction (RVEF), mediated by an increase in RV end systolic volume. RVEF improved in the stable stage but remained borderline reduced in comparison to controls $(\mathrm{p}=0.053)$.

Acute $\mathrm{CF}$ exacerbation was associated with a significant increase in myocardial ECV (control 24.8\% (22.9-26.0\%) vs. acute CF $27.6 \%(24.7-29.8 \%), \mathrm{p}=0.030)$ and $\mathrm{T} 1$ (control $1021 \pm 25 \mathrm{~ms}$ vs. acute CF $1056 \pm 31 \mathrm{~ms}, \mathrm{p}=0.008$ ). Myocardial ECV and T1 both improved in the stable period of the disease (ECV 26.2\% (25.4-27.2\%); T1 $1048 \pm 24 \mathrm{ms)}$ but remained higher than in controls. There was no difference in T2 values. Nine (90\%) CF patients exhibited nonischaemic RV insertion point LGE. There were no other focal LGE patterns observed and there was no evidence of previous myocardial infarction in any of the patients. Capillary permeability $\left(\mathrm{K}^{\text {trans }}\right)$ was non-significantly higher in acute CF compared to controls (Table 2). Figure 2 shows an example of myocardial tissue characterisation sequences.

Stable CF was associated with LV hypertrophy (LVH; LV mass: control $50 \pm 8 \mathrm{~g} / \mathrm{m}^{2}$ vs. stable CF $59 \pm 9 \mathrm{~g} / \mathrm{m}^{2}$; $\mathrm{p}=0.028$ ), which was predominantly driven by increased extracellular matrix mass (control $23.6 \pm 5.2 \mathrm{~g}$ vs. stable CF $27.5 \pm 3.41 \mathrm{~g} ; \mathrm{p}=0.006)$. Stable CF was also associated with $\mathrm{LV}$ dilatation (LV end systolic volume: control $30 \pm 6 \mathrm{ml} / \mathrm{m}^{2}$ vs. stable CF $36 \pm 5 \mathrm{ml} / \mathrm{m}^{2} ; \mathrm{p}=0.018 ; \mathrm{LV}$ 
Table 1 Patient characteristics

\begin{tabular}{|c|c|c|c|c|c|c|}
\hline Parameter & Control $(n=12)$ & Acute CF $(n=10)$ & $\begin{array}{l}\text { p value (vs. } \\
\text { control) }\end{array}$ & Stable CF $(n=9)$ & $\begin{array}{l}\mathrm{p} \text { value (vs. } \\
\text { control) }\end{array}$ & $\begin{array}{l}\text { p value (acute } \\
\text { vs. stable CF) }\end{array}$ \\
\hline \multicolumn{7}{|l|}{ Demographics } \\
\hline Age (years) & $37(27-45)$ & $28(25-36)$ & 0.129 & & & \\
\hline Gender (female) & $6(50 \%)$ & $2(20 \%)$ & 0.204 & & & \\
\hline $\operatorname{BSA}\left(\mathrm{m}^{2}\right)$ & $1.9(1.7-2.2)$ & $1.8(1.7-1.9)$ & 0.429 & $1.8(1.7-1.8)$ & 0.434 & \\
\hline Weight & $74(59-93)$ & $65(63-73)$ & 0.355 & $65(63-69)$ & 0.374 & \\
\hline \multicolumn{7}{|l|}{ CF manifestations } \\
\hline Pancreatic insufficiency & & $10(100 \%)$ & & & & \\
\hline DM & & $6(60 \%)$ & & & & \\
\hline PA colonisation & & $7(70 \%)$ & & & & \\
\hline Biliary disease & & $4(40 \%)$ & & & & \\
\hline ABPA & & $2(20 \%)$ & & & & \\
\hline \multicolumn{7}{|l|}{ Laboratory findings } \\
\hline $\mathrm{CRP}(\mathrm{mg} / \mathrm{l})$ & $1(0-2)$ & $32(14-121)$ & 0.001 & $19(7-24)$ & 0.001 & 0.058 \\
\hline $\mathrm{WBC}\left(\times 10^{9} / 1\right)$ & $5.9(4.9-7.0)$ & $12.0(10.0-15.8)$ & 0.001 & $10.3(8.6-12.4)$ & 0.001 & 0.066 \\
\hline \multicolumn{7}{|l|}{ ECG findings } \\
\hline PR duration (ms) & $140(120-170)$ & $140(140-165)$ & 0.502 & $160(130-170)$ & 0.556 & 0.705 \\
\hline QRS duration (ms) & $80(80-90)$ & $80(80-85)$ & 0.633 & $80(80-100)$ & 0.915 & 0.317 \\
\hline $\mathrm{QTc}(\mathrm{ms})$ & $394 \pm 21$ & $413 \pm 23$ & 0.079 & $414 \pm 23$ & 0.063 & 0.750 \\
\hline \multicolumn{7}{|l|}{ Spirometry } \\
\hline $\mathrm{FEV}_{1}(1 / \mathrm{s})$ & & $1.4 \pm 0.4$ & & $1.7 \pm 0.5$ & & 0.028 \\
\hline $\mathrm{FEV}_{1} \%$ & & $34.0 \pm 10.4$ & & $41.2 \pm 11.8$ & & 0.025 \\
\hline FVC (1) & & $2.6 \pm 0.6$ & & $3.1 \pm 0.4$ & & 0.026 \\
\hline FVC\% & & $51.6 \pm 10.8$ & & $62.4 \pm 5.3$ & & 0.017 \\
\hline
\end{tabular}

Data presented as mean \pm standard deviation or median (interquartile range) depending on distribution

$A B P A$ allergic bronchopulmonary aspergillosis, $B S A$ body surface area, $C F$ cystic fibrosis, $C R P$ c reactive protein, $D M$ diabetes mellitus, $F E V_{l}$ forced expiratory volume in $1 \mathrm{~s}, F E V_{l} \%$ percentage of predicted forced expiratory volume in $1 \mathrm{~s}, F V C$ forced vital capacity, $F V C \%$ percentage of predicted forced vital capacity, $W B C$ white blood cell

end diastolic volume: control $81 \pm 13 \mathrm{ml} / \mathrm{m}^{2}$ vs. stable $\mathrm{CF}$ $92 \pm 10 \mathrm{ml} / \mathrm{m}^{2} ; \mathrm{p}=0.052$ ) and $\mathrm{RV}$ dilatation (Table 2).

QTc interval was trending to be longer in both acute and stable CF, compared to controls (control $394 \pm 21 \mathrm{~ms}$ vs. acute CF $413 \pm 23 \mathrm{~ms} ; \mathrm{p}=0.079$; vs stable CF $414 \pm 23 \mathrm{~ms}$; $\mathrm{p}=0.063)$.

\section{Pulmonary MRI indices}

Both acute and stable CF were associated with significantly reduced pulmonary $\mathrm{T} 1$ (control $1266 \pm 42 \mathrm{~ms}$ vs. stable CF $1198 \pm 28 \mathrm{~ms} ; \mathrm{p}=0.003$ and vs. acute CF $1185 \pm 32 ; \mathrm{p}=0.001$; Table 3 ). Pulmonary T1 correlated strongly with $\mathrm{FEV}_{1} \%(\mathrm{r}=0.819, \mathrm{p}=0.004$; Fig. 3$)$. $\mathrm{CF}$ was also associated with significantly reduced pulmonary tissue blood flow, which was most pronounced in the upper lobes (Table 3; Fig. 4). There was a non-significant increase in pulmonary tissue blood flow during acute exacerbation, although it remained lower than in controls.

\section{Relationship between cardiac and pulmonary disease in CF}

Myocardial ECV $(\mathrm{r}=-0.726, \mathrm{p}=0.017)$ and $\mathrm{T} 1$ $(\mathrm{r}=-0.699, \mathrm{p}=0.024)$ showed strong negative correlations with $\mathrm{FEV}_{1} \%$ in acutely exacerbating CF patients (Fig. 3).

\section{Discussion}

Applying a multiparametric cardiopulmonary MRI technique to adults with $\mathrm{CF}$ demonstrates that acute respiratory exacerbations of $\mathrm{CF}$ are associated with significant acute myocardial contractile dysfunction. There is also a suggestion of myocardial oedema in the acute period which is related to pulmonary disease severity. In the stable state, $\mathrm{CF}$ patients demonstrate adverse myocardial remodelling, including systolic LV dilatation and $\mathrm{LVH}$, driven by focal replacement and diffuse interstitial myocardial fibrosis. RV 
Table 2 Cardiac MRI measurements

\begin{tabular}{|c|c|c|c|c|c|c|}
\hline Parameter & Control $(n=12)$ & Acute CF $(n=10)$ & $\mathrm{p}$ value (vs. control) & Stable CF $(n=9)$ & p value (vs. control) & $\begin{array}{l}\text { p value (acute } \\
\text { vs. stable CF) }\end{array}$ \\
\hline \multicolumn{7}{|l|}{ Left ventricle } \\
\hline LV EDV/BSA $\left(\mathrm{ml} / \mathrm{m}^{2}\right)$ & $81 \pm 13$ & $91 \pm 14$ & 0.112 & $92 \pm 10$ & 0.052 & 0.730 \\
\hline LV ESV/BSA $\left(\mathrm{ml} / \mathrm{m}^{2}\right)$ & $30 \pm 6$ & $39 \pm 7$ & 0.003 & $36 \pm 5$ & 0.018 & 0.089 \\
\hline $\operatorname{LV}$ EF (\%) & $63 \pm 5$ & $57 \pm 3$ & 0.004 & $61 \pm 5$ & 0.270 & 0.025 \\
\hline LV mass/BSA $\left(\mathrm{g} / \mathrm{m}^{2}\right)$ & $50 \pm 8$ & $58 \pm 7$ & 0.016 & $59 \pm 9$ & 0.028 & 0.885 \\
\hline \multicolumn{7}{|l|}{ Right ventricle } \\
\hline $\mathrm{RV} \operatorname{EDV} / \mathrm{BSA}\left(\mathrm{ml} / \mathrm{m}^{2}\right)$ & $84 \pm 12$ & $92 \pm 14$ & 0.169 & $94 \pm 11$ & 0.067 & 0.614 \\
\hline RV ESV/BSA (ml/m²) & $34 \pm 6$ & $45 \pm 10$ & 0.007 & $43 \pm 78$ & 0.007 & 0.842 \\
\hline RV EF (\%) & $59 \pm 6$ & $51 \pm 7$ & 0.006 & $54 \pm 6$ & 0.053 & 0.230 \\
\hline \multicolumn{7}{|l|}{ Atria } \\
\hline $\mathrm{LA}$ area/BSA $\left(\mathrm{cm}^{2} / \mathrm{m}^{2}\right)$ & $12 \pm 1$ & $12 \pm 2$ & 0.929 & $11 \pm 1$ & 0.315 & 0.048 \\
\hline $\mathrm{RA}$ area/BSA $\left(\mathrm{cm}^{2} / \mathrm{m}^{2}\right)$ & $12 \pm 2$ & $10 \pm 1$ & 0.008 & $10 \pm 1$ & 0.014 & 0.607 \\
\hline \multicolumn{7}{|c|}{ Myocardial tissue characterisation } \\
\hline $\operatorname{LGE}(\mathrm{g})$ & $0(0-0)$ & $0.92(0.53-1.35)$ & $<0.001$ & $1.04(0.79-1.09)$ & $<0.001$ & 0.953 \\
\hline LGE (\% LV mass) & $0(0-0)$ & $0.92(0.42-1.39)$ & $<0.001$ & $0.91(0.75-1.29)$ & $<0.001$ & 0.953 \\
\hline $\mathrm{T} 1$ (ms) & $1021 \pm 25$ & $1056 \pm 31$ & 0.008 & $1048 \pm 24$ & 0.027 & 0.246 \\
\hline $\mathrm{T} 2(\mathrm{~ms})$ & $48 \pm 2$ & $49 \pm 2$ & 0.697 & $48 \pm 2$ & 0.666 & 0.344 \\
\hline $\operatorname{ECV}(\%)$ & $24.8(22.9-26.0)$ & $27.6(24.7-29.8)$ & 0.030 & $26.2(25.4-27.2)$ & 0.047 & 0.515 \\
\hline $\begin{array}{l}\text { Extracellular matrix } \\
\text { mass }(\mathrm{g})\end{array}$ & $23.6 \pm 5.2$ & $28.7 \pm 4.9$ & 0.027 & $27.5 \pm 3.41$ & 0.006 & 0.571 \\
\hline Cellular mass (g) & $72.6 \pm 18.5$ & $76.7 \pm 11.5$ & 0.56 & $77.83 \pm 13.8$ & 0.49 & 0.62 \\
\hline $\mathrm{K}^{\text {trans }}\left(\min ^{-1}\right)$ & $0.33 \pm 0.05^{*}$ & $0.39 \pm 0.11$ & 0.298 & $0.37 \pm 0.05$ & 0.192 & 0.617 \\
\hline
\end{tabular}

Data presented as mean \pm standard deviation or median (interquartile range) depending on distribution. $*_{\mathrm{n}}=5$

$E C V$ extracellular volume fraction, $E D V$ end diastolic volume, $E F$ ejection fraction, $E S V$ end systolic volume, $K^{\text {trans }}$ transfer constant, $L A$ left atrium, $L G E$ late gadolinium enhancement, $L V$ left ventricle, $R A$ right atrium, $R V$ right ventricle. Other abbreviations as per Table 1

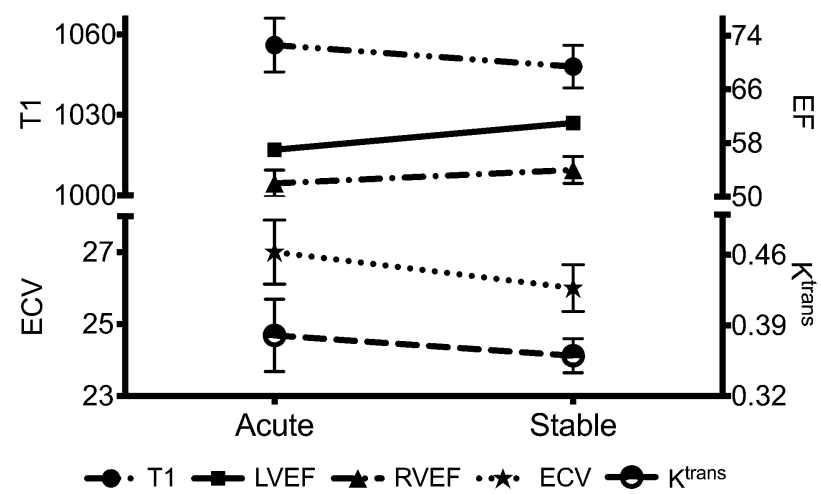

Fig. 1 Changes in cardiac structure and function between acute respiratory exacerbation and stable period. There was a reduction in myocardial capillary permeability $\left(\mathrm{K}^{\text {trans }} ; \mathrm{min}^{-1}\right)$ between acute respiratory exacerbation and stable stage. Likely as a result, myocardial extracellular volume $(\mathrm{ECV} ; \%)$ and $\mathrm{T} 1$ relaxation $(\mathrm{ms})$ time both reduced, in keeping with a reduction in myocardial oedema. In turn, left and right ventricular contractile function improved ( $L V E F$ left ventricular ejection fraction; \%; RVEF right ventricular ejection fraction; \%) dilatation and dysfunction were also evident despite no clinical evidence of cor pulmonale.

It is increasingly recognised that lung diseases are associated with cardiovascular diseases, independent of shared risk factors, and that, in turn, cardiovascular diseases independently contribute to all-cause mortality in patients with lung conditions $[19,20]$. The association is particularly strong for heart failure and is independent of the increased risk of coronary artery disease [20]. The mechanisms underlying these associations are unclear, but myocardial inflammation occurring as part of systemic inflammation, with subsequent interstitial myocardial fibrosis, leading to mechanical, electrical and vasomotor myocardial dysfunction, is a widely held hypothesis [21]. However, evidence for this is limited, in part because existing methods to investigate these disease mechanisms, such as circulating markers of inflammation and fibrosis, are not organ specific.

The cardiopulmonary MRI technology provides direct and simultaneous interrogation of cardiac and pulmonary pathophysiology, and their relationship, including mechanical function, inflammation, fibrosis and blood flow characteristics. Indeed, the myocardial injury identified using the MRI protocol was not evident from circulating biomarkers 


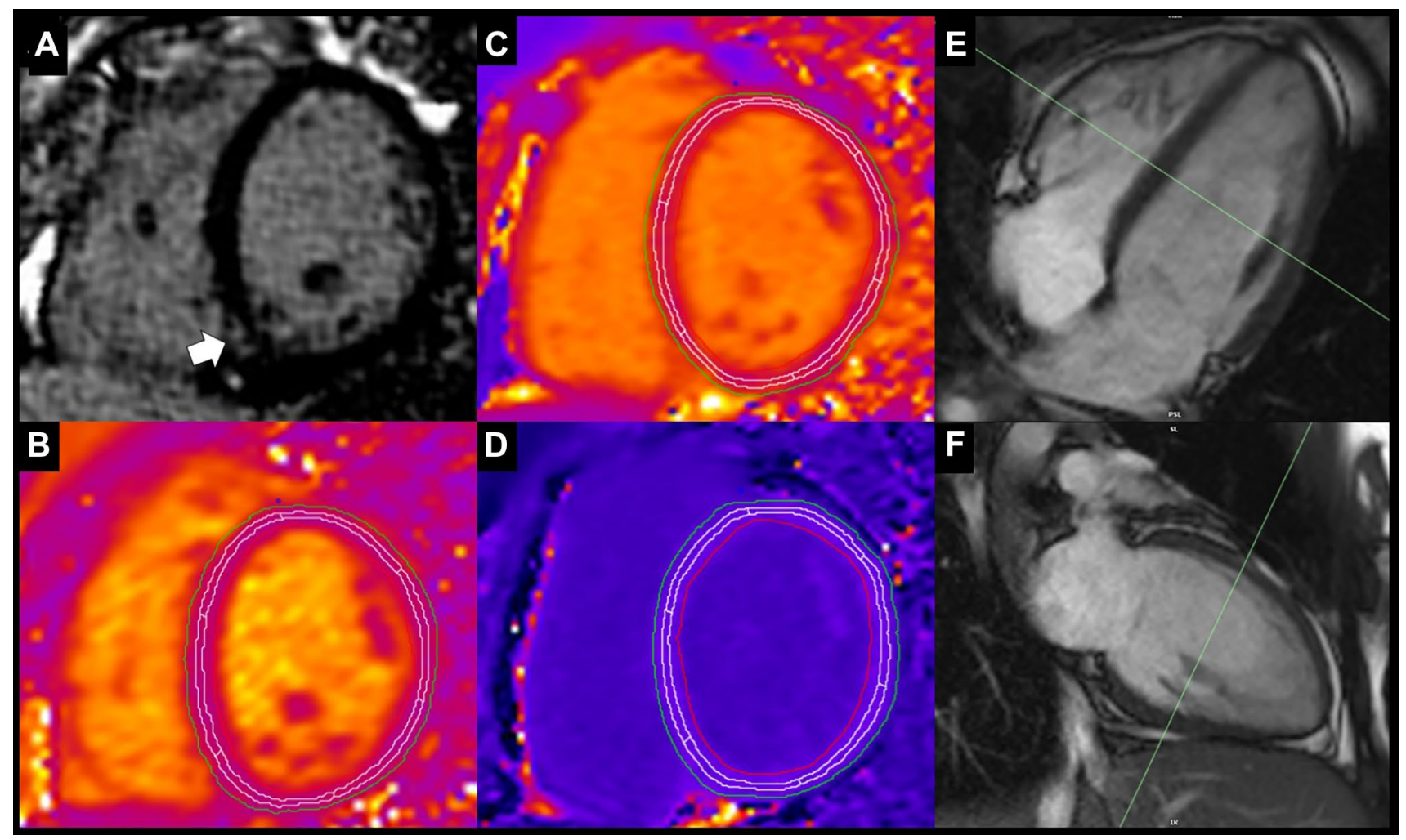

Fig. 2 Myocardial tissue characterisation in cystic fibrosis. Representative endocardial and epicardial regions of interest for T1 and T2 mapping are shown. Mid third of the left ventricle (LV) wall was used for analysis. A Inferior right ventricle insertion point late enhance- ment (arrow). B Mid LV T2 mapping (T2=46 ms). C Mid LV native T1 mapping $(\mathrm{T} 1=1088 \mathrm{~ms})$. D Mid LV post contrast T1 mapping (post contrast $\mathrm{T} 1=588 \mathrm{~ms}$ ). E, $\mathbf{F}$ Long axis cine imaging showing the position of mid LV short axis parametric mapping slices

Table 3 Pulmonary MRI measurements

\begin{tabular}{|c|c|c|c|c|c|c|}
\hline Parameter & Controls & Acute CF $(n=10)$ & $\begin{array}{l}\mathrm{p} \text { value (vs. } \\
\text { control) }\end{array}$ & Stable CF $(n=9)$ & $\begin{array}{l}\mathrm{p} \text { value (vs. } \\
\text { control) }\end{array}$ & $\begin{array}{l}\text { p value (acute } \\
\text { vs. stable CF) }\end{array}$ \\
\hline \multicolumn{7}{|c|}{ Pulmonary tissue characterisation } \\
\hline $\mathrm{T} 1(\mathrm{~ms})$ & $1266 \pm 42^{\dagger}$ & $1185 \pm 32$ & 0.001 & $1198 \pm 28$ & 0.003 & 0.643 \\
\hline $\operatorname{Ve}(\%)$ & $24.5(14.1-29.4) *$ & $26.4(21.0-29.5)$ & 0.462 & $24.1(21.8-29.4)$ & 0.641 & 0.767 \\
\hline $\mathrm{K}^{\text {trans }}\left(\min ^{-1}\right)$ & $0.27 \pm 0.07 *$ & $0.23 \pm 0.07$ & 0.399 & $0.26 \pm 0.07$ & 0.791 & 0.445 \\
\hline \multicolumn{7}{|c|}{ Pulmonary tissue blood flow } \\
\hline Both lungs & $2.84(1.91-3.39)^{*}$ & $1.63(1.45-1.89)$ & 0.027 & $1.51(1.02-1.71)$ & 0.053 & 0.594 \\
\hline Right upper lobe & $2.84(1.71-3.01)^{*}$ & $0.99(0.73-1.30)$ & 0.007 & $0.83(0.57-1.88)$ & 0.014 & 0.260 \\
\hline Right lower lobe & $3.33(1.99-4.00)^{*}$ & $1.92(1.61-2.16)$ & 0.066 & $1.67(1.27-3.43)$ & 0.125 & 0.441 \\
\hline Right middle lobe & $2.72(1.82-3.81)^{*}$ & $1.93(1.34-2.46)$ & 0.142 & $1.51(0.94-2.58)$ & 0.072 & 0.859 \\
\hline Left upper lobe & $1.94(1.67-3.43)^{*}$ & $1.42(1.15-2.03)$ & 0.053 & $1.09(0.78-1.68)$ & 0.028 & 0.594 \\
\hline Left lower lobe & $2.47(1.93-4.00)^{*}$ & $2.46(1.81-2.58)$ & 0.463 & $1.87(1.41-2.68)$ & 0.125 & 0.374 \\
\hline
\end{tabular}

Data presented as mean \pm standard deviation or median (interquartile range) depending on distribution. Measurements are averaged for both lungs unless stated otherwise. Pulmonary blood flow units are $\mathrm{ml} \mathrm{blood} / \mathrm{ml} \mathrm{tissue} / \mathrm{min}$

${ }^{\Delta} \mathrm{n}=9 ;{ }^{\dagger} \mathrm{n}=8 ; * \mathrm{n}=5 ; \mathrm{Ve}$ - pulmonary extracellular volume fraction. Other abbreviations as per Table 2

or ECG. The accuracy and reproducibility of MRI mean that relatively small sample sizes are often sufficient [22]. Echocardiography studies in CF have produced conflicting findings, in part because of the high variability of echocardiographic measurements [3-5]. Ours is the first study to apply cardiac MRI in CF. 

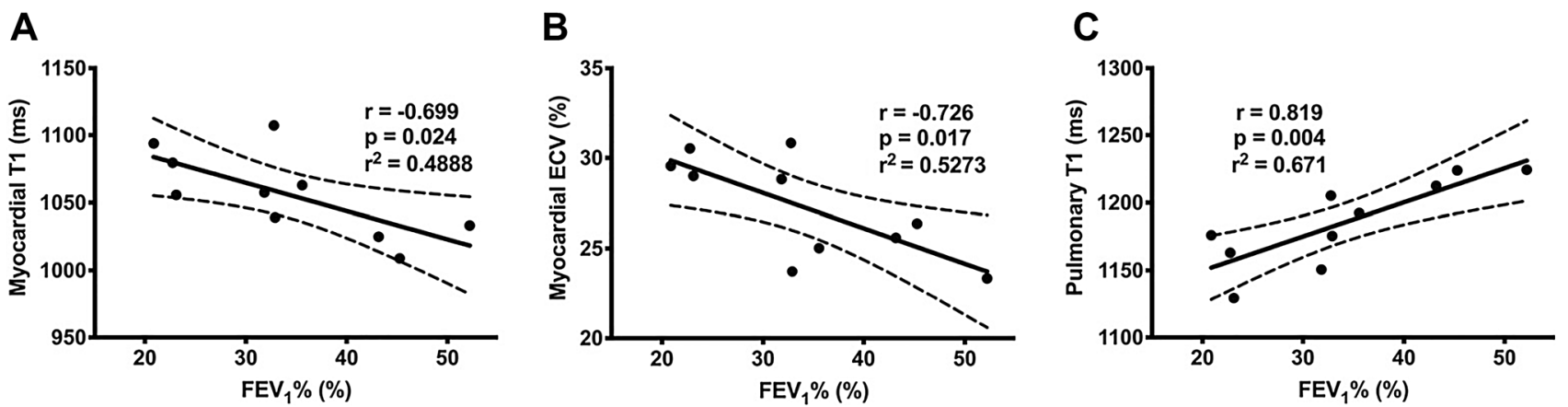

Fig. 3 Relationship between myocardial and pulmonary tissue characteristics and percentage of predicted normal forced expiratory volume in one second $\left(\mathrm{FEV}_{1} \%\right)$. In acutely exacerbating cystic fibrosis patients, a myocardial $\mathrm{T} 1$ relaxation time and $\mathbf{b}$ myocardial extracel- lular volume (ECV), both indicative of myocardial oedema, showed strong negative correlations with $\mathrm{FEV}_{1} \%$. c Pulmonary $\mathrm{T} 1$ relaxation time was strongly correlated with $\mathrm{FEV}_{1} \%$
Fig. 4 Pulmonary tissue blood flow maps. a, b Right lung in patients with cystic fibrosis. There is a visible reduction in pulmonary tissue blood flow in right upper lobes (arrows). c, d Right lung in healthy controls
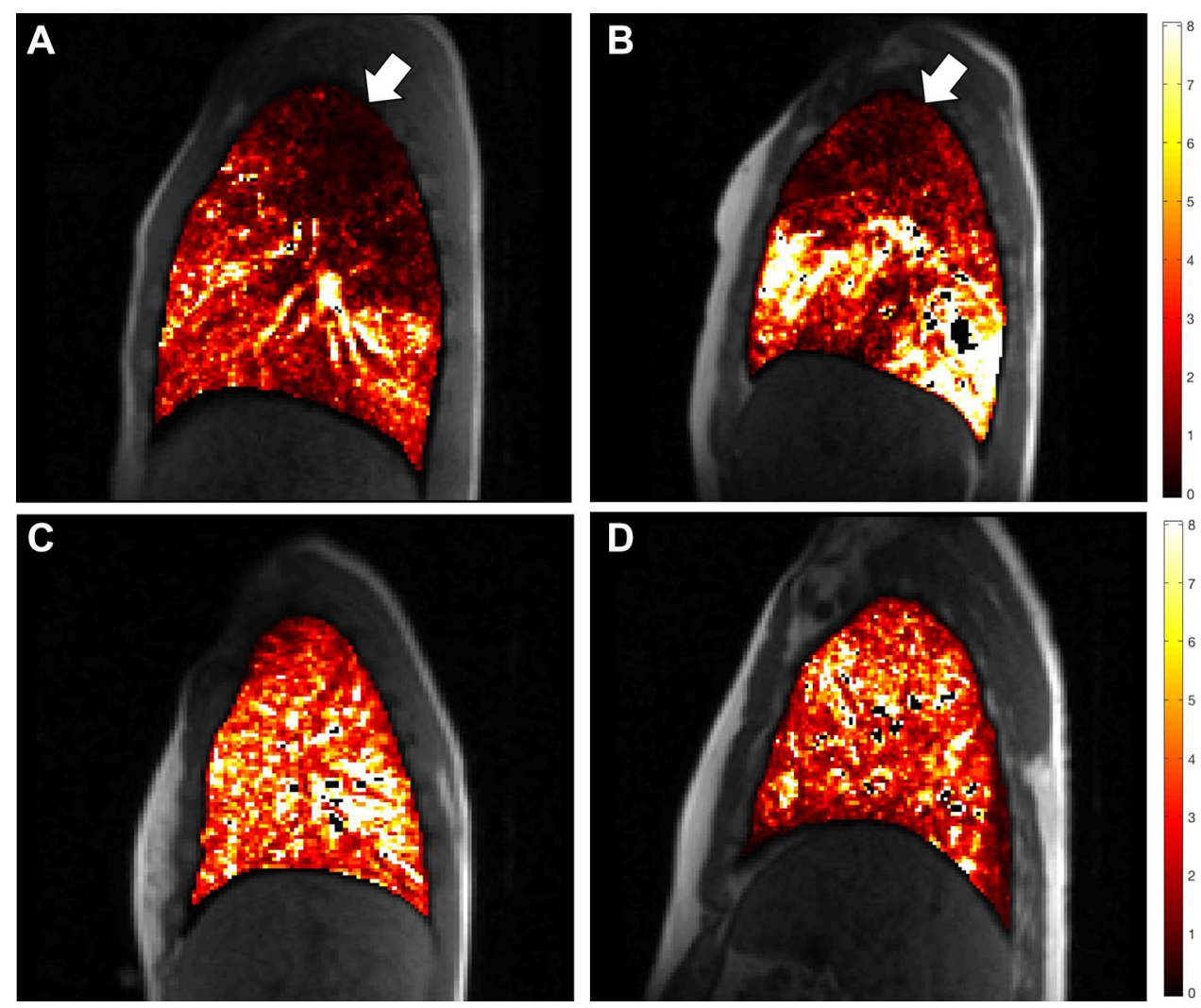

Preclinical studies demonstrate that loss of CFTR function has a primary myocardial effect. CFTR is important for regulation of resting cardiomyocyte membrane potential and action potential duration [2]. Acute inhibition of CFTR leads to a transient decrease in cardiomyocyte contractility, increase in intracellular calcium concentration and inhibition of protein kinase A activity [23]. In order to normalise contractility, $\mathrm{Ca} 2 \%$ calmodulin-dependent kinase II (CaMKII) and $\mathrm{Ca}^{2+}$-activated chloride channels are chronically activated [23]. Whilst this is sufficient to maintain resting contractility, response to $\beta$-adrenergic stimulation is impaired [24]. Furthermore, CaMKII upregulation is associated with LV dilatation, LVH and QT prolongation $[25,26]$. The LV hypertrophy observed in preclinical models is independent of other organ involvement.

Our findings, which are remarkably consistent with the preclinical findings, provide novel insight into cardiac involvement in $\mathrm{CF}$ in humans. Specifically, we demonstrate that in the stable state, $\mathrm{CF}$ is associated with systolic LV dilatation, LVH and QT prolongation. Furthermore, in keeping with the impaired cardiomyocyte stress response demonstrated in vitro, we show that acute respiratory 
exacerbations of $\mathrm{CF}$ are associated with a fall in LVEF (also see below).

It has been hypothesised that $\mathrm{CF}$ is associated with myocardial fibrosis [27], but other than autopsy reports of myocardial necrosis and fibrosis following infant sudden death, there is little available evidence [28]. MRIderived ECV quantifies myocardial extracellular matrix volume and in non-acute situations is a robust measure of myocardial fibrosis, having been extensively validated histologically [10]. Myocardial T1 is a surrogate of ECV, however, the values are more scanner- and sequence dependent compared to ECV. Myocardial LGE provides a robust identification of focal replacement fibrosis [9]. Using these techniques we show, for the first time, that stable $\mathrm{CF}$ is associated with diffuse interstitial and replacement fibrosis. Indeed, we demonstrate that the identified LVH in CF is predominantly driven by myocardial fibrosis rather than cellular hypertrophy. Hypothesised causes of myocardial fibrosis in CF include recurrent inflammation (see below), chronic hypoxia, activation of the renin-angiotensin-aldosterone system and hyperglycaemia [27]. The impaired LV diastolic function and strain observed in some echocardiographic studies may be secondary to fibrosis $[4,5]$. Myocardial fibrosis is consistently associated with adverse outcome in other conditions [29] and investigation of the relationship between myocardial fibrosis and prognosis in $\mathrm{CF}$ is required.

In addition to chronic myocardial manifestations, this study demonstrates that acute respiratory CF exacerbation is associated with acute myocardial contractile dysfunction. There was a significant fall in LVEF seen during an acute exacerbation, which normalised in the chronic period. Myocardial ECV and T1 are also reflective of myocardial water content and, in the appropriate clinical context, are markers of myocardial oedema. ECV, T1, and $\mathrm{K}^{\text {trans }}$ were all higher in acute $\mathrm{CF}$ compared to stable $\mathrm{CF}$, although the differences were not statistically significant. This is likely as a result of the small sample size. T2 values were no different between acute and chronic CF. Although T2 was shown to be highly specific for detecting myocardial oedema, it has a lower sensitivity and lower diagnostic accuracy compared to T1 mapping $[30,31]$ which could explain why T1 mapping detected changes that T2 mapping did not. Despite the lack of statistical significance, the behaviour of ECV, T1 and $\mathrm{K}^{\text {trans }}$ indices in acute and chronic $\mathrm{CF}$ suggests a presence of myocardial oedema at the time of pulmonary exacerbation which then possibly resolves in the chronic period. This finding provides a strong foundation for future, larger studies, appropriately powered to detect the changes in MRI-derived oedema indices. The myocardial manifestations were observed despite the acute MRI scan taking place 12 days post-admission therefore findings should be more apparent if scanning takes place earlier.
Putting the findings together, we hypothesise that an acute respiratory $\mathrm{CF}$ exacerbation leads to systemic inflammation, as part of which there is increased myocardial capillary permeability, leading to myocardial oedema. As a result of the oedema, and the aforementioned impaired contractile response of cardiomyocytes to stress, LV function acutely declines. Recurrent episodes of myocardial inflammation may contribute to fibrosis. Larger studies involving histological validation will be required to confirm this hypothesis.

It is important to acknowledge alternative and confounding mechanisms that could contribute to contractile dysfunction in CF patients. Increased large artery stiffness is present in CF patients and is weakly correlated to inflammatory status [32]. Increased afterload during an acute exacerbation could contribute to an impairment of systolic function. CF patients were also shown to have reduced nitric oxide bioavailability and endothelial dysfunction [33]. Although premature coronary artery disease in major epicardial vessels is not usually part of CF disease spectrum [34] and there was no evidence of previous myocardial infarction in any of our participants, coronary microvascular dysfunction was shown to contribute to contractile dysfunction in other conditions [35]. Additionally, hypoxia accompanying an acute CF exacerbation could further worsen endothelial dysfunction [36]. Coronary assessment was not part of the study (see "Limitations" section). Finally, LV contractility can be affected by chronic activation of (CaMKII) and $\mathrm{Ca}^{2+}$-activated chloride channels, especially in the presence of increased $\beta$-adrenergic stimulation as described above [24].

$\mathrm{CF}$ was associated with impaired pulmonary tissue blood flow, which was most marked in the upper lobes, in keeping with the known pattern of disease progression [37]. Pulmonary tissue blood flow increased during acute exacerbation in comparison to stable $\mathrm{CF}$, which may reflect relative hyperaemia as part of the inflammatory response, but the difference was not significant, and it remained substantially lower than in controls. In keeping with previous studies pulmonary $\mathrm{T} 1$ was reduced, likely reflecting parenchymal loss, the severity of which correlated strongly with FEV1\% $[13,37]$. Capillary permeability and pulmonary extracellular volume measurements are dependent on GBCA delivery and mixing, thus were likely underestimated as a result of low pulmonary tissue perfusion.

The severity of the myocardial injury, specifically myocardial oedema, showed a strong association with airflow limitation. This is a key finding and demonstrates the relationship between heart and lung pathophysiology. $\mathrm{FEV}_{1}$ is perceived to be the strongest prognostic indicator in $\mathrm{CF}$ because it indicates the severity of lung disease, however, it may be that in addition, $\mathrm{FEV}_{1}$ is a marker of the severity of myocardial injury. Further investigation is required.

There were no associations between other heart and lung measurements. Indeed, despite improvements in symptoms, 
spirometry and circulating inflammatory markers between the acute and stable assessments, there were no significant improvements in lung MRI indices. This may indicate that the lung disease in this cohort was at an advanced, essentially irreversible stage, whereas the myocardium was relatively less affected and thus acute-on-chronic injury was evident. Application of the protocol in patients with less severe lung disease would be interesting. The lack of association between heart and lung disease suggests myocardial disease may occur independently of pulmonary disease and indicates the requirement for dedicated cardiac evaluation.

\section{Limitations}

The number of patients included was small, but despite this the results were consistent with preclinical findings and achieved statistical significance. The impact of acute respiratory exacerbation on myocardium may be underestimated as the requirement for patients to hold their breath during many of the MRI acquisitions made it impossible to perform the acute MRI closer to the admission date. Whilst the relationship between ECV and native $\mathrm{T} 1$ and histological myocardial fibrosis has been demonstrated in multiple previous studies, histological validation was not performed in the current study, and the relationship between ECV and T1 mapping and histological myocardial fibrosis demonstrated in the previous studies may not necessarily extrapolate to the current study $[10,18]$. Anatomical or functional coronary imaging was not part of the study. Although premature coronary artery disease is not usually considered part of CF spectrum and none of the patients had evidence of myocardial infarction on LGE imaging, undiagnosed coronary artery disease could contribute to contractile dysfunction.

\section{Conclusions}

Cardiopulmonary MRI technology allows for simultaneous interrogation of cardiac and pulmonary structure and function, and evaluates their relationship. We demonstrate that stable CF is associated with adverse myocardial remodelling, including systolic LV dilatation and LV hypertrophy, driven by focal replacement and diffuse interstitial myocardial fibrosis. In addition, we demonstrate that acute respiratory exacerbation of $\mathrm{CF}$ is associated with acute myocardial contractile dysfunction. There is also a suggestion of myocardial oedema in the acute period, the severity of which is related to pulmonary dysfunction.
Supplementary Information The online version contains supplementary material available at https://doi.org/10.1007/s10554-021-02496-6.

Acknowledgements The authors would like to express their gratitude to Siemens Healthcare for technical support.

Author contributions JL and CAM designed the study, had full access to all the data in the study and take responsibility for the integrity of the work as a whole. All authors contributed towards data analysis, drafting and revising the paper and agree to be accountable for all aspects of the work.

Funding Dr Lagan is funded by a Clinical Research Training Fellowship from the British Heart Foundation (FS/17/47/32805). Dr Miller is funded by a Clinician Scientist Award (CS-2015-15-003) from the National Institute for Health Research. The work was also supported in part by a British Heart Foundation Accelerator award to The University of Manchester (AA/18/4/34221). The views expressed in this publication are those of the authors and not necessarily those of the NHS, the National Institute for Health Research or the Department of Health. None of these funding bodies had a role in study design, data collection, analysis or interpretation or writing of the manuscript.

Data availability The datasets generated and/or analysed during the current study are available from the corresponding author on reasonable request.

\section{Declarations}

Conflict of interest J.L. reports a grant from British Heart Foundation (Clinical Research Training Fellowship). C.A.M. reports grants from National Institute for Health Research, UK and from British Heart Foundation during the conduct of the study and research support from Amicus Therapeutics, Guerbet Laboratories Limited, Roche and Univar Solutions B.V. outside of the submitted work. C.A.M. has served on an advisory board for Novartis, Boehringer Ingelheim and Lilly Alliance, and AstraZeneca, and serves as an advisor for HAYA therapeutics and PureTech Health. J.H.N., J.B., C.F., C.P., D.C., E.B.S., M.S. and R.B.T. have nothing to disclose. The sponsor and funders had no role in study design, data collection and analysis, decision to publish or preparation of the manuscript.

Ethical approval The Research Ethics Committee North West-Greater Manchester South approved the study (reference number 14/NW/1088). Written informed consent was obtained from all participants. The work was conducted according to the Helsinki Declaration.

Consent for publication Not applicable.

Open Access This article is licensed under a Creative Commons Attribution 4.0 International License, which permits use, sharing, adaptation, distribution and reproduction in any medium or format, as long as you give appropriate credit to the original author(s) and the source, provide a link to the Creative Commons licence, and indicate if changes were made. The images or other third party material in this article are included in the article's Creative Commons licence, unless indicated otherwise in a credit line to the material. If material is not included in the article's Creative Commons licence and your intended use is not permitted by statutory regulation or exceeds the permitted use, you will need to obtain permission directly from the copyright holder. To view a copy of this licence, visit http://creativecommons.org/licenses/by/4.0/. 


\section{References}

1. Corriveau S, Sykes J, Stephenson AL (2018) Cystic fibrosis survival: the changing epidemiology. Curr Opin Pulm Med 24(6):574-578

2. Warth JD, Collier ML, Hart P, Geary Y, Gelband CH, Chapman T et al (1996) CFTR chloride channels in human and simian heart. Cardiovasc Res 31(4):615-624

3. Giacchi V, Rotolo N, Amato B, Di Dio G, Betta P, La Rosa M et al (2015) Heart involvement in children and adults with cystic fibrosis: correlation with pulmonary indexes and inflammation markers. Heart Lung Circ 24(10): 1002-1010

4. Eising JB, van der Ent CK, Teske AJ, Vanderschuren MM, Uiterwaal C, Meijboom FJ (2018) Young patients with cystic fibrosis demonstrate subtle alterations of the cardiovascular system. J Cyst Fibros 17(5):643-649

5. Labombarda F, Pellissier A, Ellafi M, Creveuil C, Ribault V, Laurans M et al (2011) Myocardial strain assessment in cystic fibrosis. J Am Soc Echocardiogr 24(9):1037-1045

6. Quintana-Gallego E, Ruiz-Ramos M, Delgado-Pecellin I, Calero C, Soriano JB, Lopez-Campos JL (2016) Mortality from cystic fibrosis in Europe: 1994-2010. Pediatr Pulmonol 51(2):133-142

7. Lagan J, Naish JH, Fortune C, Bradley J, Clark D, Niven R et al (2021) Myocardial involvement in eosinophilic granulomatosis with polyangiitis evaluated with cardiopulmonary magnetic resonance. Int J Cardiovasc Imaging 37(4):1371-1381

8. Schulz-Menger J, Bluemke DA, Bremerich J, Flamm SD, Fogel MA, Friedrich MG et al (2013) Standardized image interpretation and post processing in cardiovascular magnetic resonance: Society for Cardiovascular Magnetic Resonance (SCMR) board of trustees task force on standardized post processing. J Cardiovasc Magn Reson 15(1):35

9. Moravsky G, Ofek E, Rakowski H, Butany J, Williams L, RalphEdwards A et al (2013) Myocardial fibrosis in hypertrophic cardiomyopathy: accurate reflection of histopathological findings by CMR. JACC Cardiovasc Imaging 6(5):587-596

10. Messroghli DR, Moon JC, Ferreira VM, Grosse-Wortmann L, He T, Kellman P et al (2017) Clinical recommendations for cardiovascular magnetic resonance mapping of $\mathrm{T} 1, \mathrm{~T} 2, \mathrm{~T} 2 *$ and extracellular volume: A consensus statement by the Society for Cardiovascular Magnetic Resonance (SCMR) endorsed by the European Association for Cardiovascular Imaging (EACVI). J Cardiovasc Magn Reson 19(1):75

11. Cutillo AG, Chan PH, Ailion DC, Watanabe S, Rao NV, Hansen CB et al (2002) Characterization of bleomycin lung injury by nuclear magnetic resonance: correlation between NMR relaxation times and lung water and collagen content. Magn Reson Med 47(2):246-256

12. Stadler A, Jakob PM, Griswold M, Stiebellehner L, Barth M, Bankier AA (2008) T1 mapping of the entire lung parenchyma: Influence of respiratory phase and correlation to lung function test results in patients with diffuse lung disease. Magn Reson Med 59(1):96-101

13. Alamidi DF, Morgan AR, Hubbard Cristinacce PL, Nordenmark LH, Hockings PD, Lagerstrand KM et al (2016) COPD patients have short lung magnetic resonance T1 relaxation time. COPD 13(2):153-159

14. Naish JH, Kershaw LE, Buckley DL, Jackson A, Waterton JC, Parker GJM (2009) Modeling of contrast agent kinetics in the lung using T1-weighted dynamic contrast-enhanced MRI. Magn Reson Med 61(6):1507-1514

15. Hueper K, Parikh MA, Prince MR, Schoenfeld C, Liu C, Bluemke DA et al (2013) Quantitative and semiquantitative measures of regional pulmonary microvascular perfusion by magnetic resonance imaging and their relationships to global lung perfusion and lung diffusing capacity: the multiethnic study of atherosclerosis chronic obstructive pulmonary disease study. Invest Radiol 48(4):223-230
16. Claesson-Welsh L (2015) Vascular permeability-the essentials. Upsala J Med Sci 120(3):135-143

17. Fuchs HJ, Borowitz DS, Christiansen DH, Morris EM, Nash ML, Ramsey BW et al (1994) Effect of aerosolized recombinant human DNase on exacerbations of respiratory symptoms and on pulmonary function in patients with cystic fibrosis The Pulmozyme Study Group. N Engl J Med 331(10):637-642

18. Miller CA, Naish JH, Bishop P, Coutts G, Clark D, Zhao S et al (2013) Comprehensive validation of cardiovascular magnetic resonance techniques for the assessment of myocardial extracellular volume. Circ Cardiovasc Imaging 6(3):373-383

19. Carter P, Lagan J, Fortune C, Bhatt DL, Vestbo J, Niven R et al (2019) Association of Cardiovascular Disease With Respiratory Disease. J Am Coll Cardiol 73(17):2166-2177

20. Lagan J, Schelbert EB, Naish JH, Vestbo J, Fortune C, Bradley J et al (2021) Mechanisms Underlying the Association of Chronic Obstructive Pulmonary Disease With Heart Failure. JACC Cardiovasc Imaging 14(10):1963-1973

21. Paulus WJ, Tschöpe C (2013) A novel paradigm for heart failure with preserved ejection fraction: comorbidities drive myocardial dysfunction and remodeling through coronary microvascular endothelial inflammation. J Am Coll Cardiol 62(4):263-271

22. Bellenger NG, Davies LC, Francis JM, Coats AJ, Pennell DJ (2000) Reduction in sample size for studies of remodeling in heart failure by the use of cardiovascular magnetic resonance. J Cardiovasc Magn Reson 2(4):271-278

23. Sellers ZM, De Arcangelis V, Xiang Y, Best PM (2010) Cardiomyocytes with disrupted CFTR function require CaMKII and $\mathrm{Ca}(2+)-$ activated $\mathrm{Cl}(-)$ channel activity to maintain contraction rate. $\mathrm{J}$ Physiol 588(Pt 13):2417-2429

24. Sellers ZM, Kovacs A, Weinheimer CJ, Best PM (2013) Left ventricular and aortic dysfunction in cystic fibrosis mice. J Cyst Fibros 12(5):517-524

25. Zhang T, Johnson EN, Gu Y, Morissette MR, Sah VP, Gigena MS et al (2002) The cardiac-specific nuclear delta(B) isoform of Ca2+l calmodulin-dependent protein kinase II induces hypertrophy and dilated cardiomyopathy associated with increased protein phosphatase 2A activity. J Biol Chem 277(2):1261-1267

26. Bers DM (2008) Calcium cycling and signaling in cardiac myocytes. Annu Rev Physiol 70(1):23-49

27. Labombarda F, Saloux E, Brouard J, Bergot E, Milliez P (2016) Heart involvement in cystic fibrosis: a specific cystic fibrosis-related myocardial changes? Respir Med 118:31-38

28. Zebrak J, Skuza B, Pogorzelski A, Ligarska R, Kopytko E, Pawlik J et al (2000) Partial CFTR genotyping and characterisation of cystic fibrosis patients with myocardial fibrosis and necrosis. Clin Genet 57(1):56-60

29. Schelbert EB, Fridman Y, Wong TC, Abu Daya H, Piehler KM, Kadakkal A et al (2017) Temporal relation between myocardial fibrosis and heart failure with preserved ejection fraction: association with baseline disease severity and subsequent outcome. JAMA Cardiol 2(9):995-1006

30. Luetkens JA, Homsi R, Sprinkart AM, Doerner J, Dabir D, Kuetting DL et al (2016) Incremental value of quantitative CMR including parametric mapping for the diagnosis of acute myocarditis. Eur Heart J Cardiovasc Imaging 17:154-161

31. Lagan J, Schmitt M, Miller CA (2018) Clinical applications of multi-parametric CMR in myocarditis and systemic inflammatory diseases. Int J Cardiovasc Imaging 34(1):35-54

32. Hull JH, Garrod R, Ho TB, Knight RK, Cockcroft JR, Shale DJ et al (2009) Increased augmentation index in patients with cystic fibrosis. Eur Respir J 34(6): 1322-1328

33. Poore S, Berry B, Eidson D, McKie KT, Harris RA (2013) Evidence of vascular endothelial dysfunction in young patients with cystic fibrosis. Chest 143(4):939-945 
34. Skolnik K, Levy RD, Wilcox PG, Quon BS (2016) Coronary artery disease in cystic fibrosis: an emerging concern? J Cyst Fibros 15(6):e70-e71

35. Bajaj NS, Singh A, Zhou W, Gupta A, Fujikura K, Byrne C et al (2020) Coronary microvascular dysfunction, left ventricular remodeling, and clinical outcomes in patients with chronic kidney impairment. Circulation 141(1):21-33

36. Konijnenberg LSF, Damman P, Duncker DJ, Kloner RA, Nijveldt R, van Geuns R-JM et al (2020) Pathophysiology and diagnosis of coronary microvascular dysfunction in ST-elevation myocardial infarction. Cardiovasc Res 116(4):787-805
37. Donnola SB, Dasenbrook EC, Weaver D, Lu L, Gupta K, Prabhakaran A et al (2017) Preliminary comparison of normalized T1 and non-contrast perfusion MRI assessments of regional lung disease in cystic fibrosis patients. J Cyst Fibros 16(2):283-290

Publisher's Note Springer Nature remains neutral with regard to jurisdictional claims in published maps and institutional affiliations. 